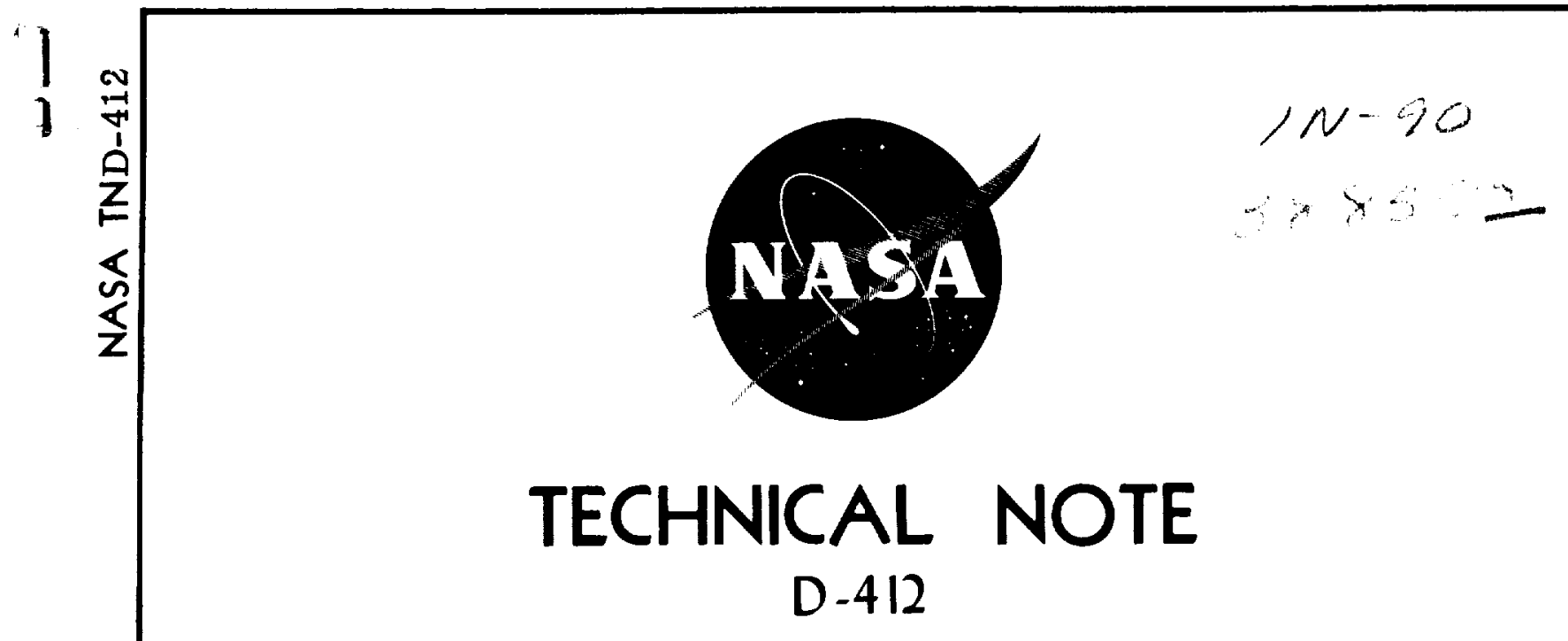

THE FLUX AND ENERGY SPECTRA OF THE PROTONS IN THE INNER VAN ALLEN BELT

\author{
John E. Naugle \\ and \\ Donald A. Kniffen
}

Goddard Space Flight Center

Greenbelt, Maryland

NATIONAL AERONAUTICS AND SPACE ADMINISTRATION WASHINGTON 


\section{THE FLUX AND ENERGY SPECTRA OF THE PROTONS IN THE INNER VAN ALLEN BELT}

by

John E. Naugle and Donald A. Kniffen

Goddard Space Flight Center

\section{SUMMARY}

A cylindrical stack of G-5 nuclear emulsions housed in the payload section of a four-stage research rocket was flown into the northern edge of the inner Van Allen belt on September 19, 1960. The experimental design permitted, for the first time, measurements of the particle fluxes and energy spectra as functions of position along the rocket trajectory. Eight points along the trajectory have been selected for analysis. Results are presented herein for three of these points, and they are discussed in the light of various theories on the trapped radiation. 
9
7
1

\section{CONTENTS}

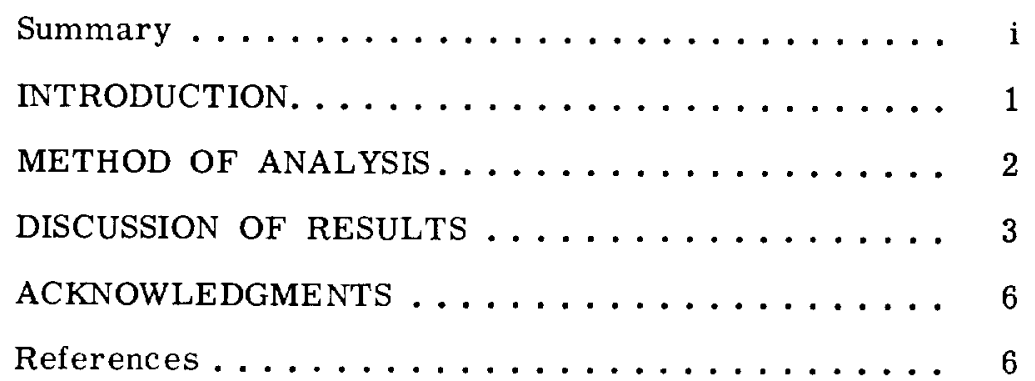




\section{THE FLUX AND ENERGY SPECTRA OF THE PROTONS IN THE INNER VAN ALLEN BELT}

by

John E. Naugle and Donald A. Kniffen

Goddord Space Flight Center

\section{INTRODUCTION}

Prior to the experiment described herein, the proton energy spectrum in the inner Van Allen radiation belt had been measured (References 1 and 2) only with nuclear emulsions which integrated the results over the entire flight paths of missiles launched from Cape Canaveral, Florida. On September 19, 1960, a research rocket especially equipped for the exposure and recovery of nuclear emulsions was flown along a trajectory closely corresponding to a magnetic meridian, obtaining the first measurements of the flux and energy spectra of these trapped protons as functions of position in the belt.

The rocket payload was designed to permit a four-inch cylinder to be extended through the front end of the nose cone, thereby reducing to a minimum the amount of material surrounding the emulsion container. Time resolution of the spectrum was obtained by exposing sections of a cylindrical emulsion stack to the ambient radiation through a port in the tungsten emulsion container (Figure 1). A point on the periphery of the stack was behind the port for approximately 80 seconds. During the rest of the flight the point was shielded by $30.6 \mathrm{gm} / \mathrm{cm}^{2}$ of tungsten. Therefore, the track population at such a point consists of particles which came through the port during the 80 -second exposure plus a background of particles which passed through the tungsten during the remaining 900 seconds of flight. A portion of the stack which did not pass behind the port provided a measure of the background. Protons with energies $E \geq 8 \mathrm{Mev}$ were able to penetrate the aluminum port cover and be detected, whereas only protons with $\mathrm{E} \geq 145 \mathrm{Mev}$ were recorded in the emulsion after passing through the tungsten shield.

\section{METHOD OF ANALYSIS}

The payload trajectory is shown in Figure 2. Numbered points along the trajectory indicate the locations where the corresponding points on the stack were behind the port. Table 1 gives the pertinent data regarding three of the eight points selected for analysis. 


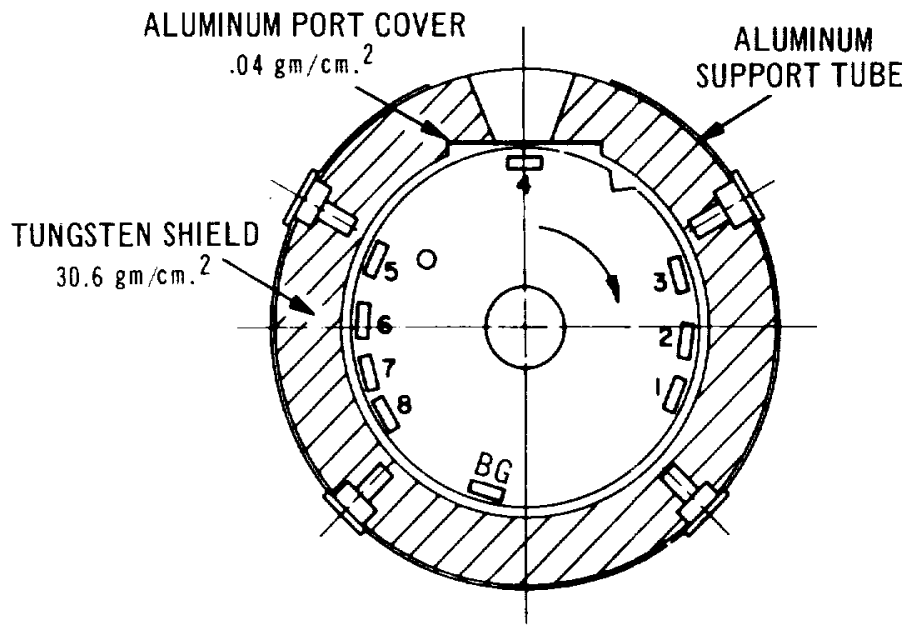

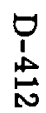

Figure 1 - 25 circular $G-5$ and $G-0$ nu:lear emulsions were contained in a tungsten cassett 6 ; the numbered points around the periphery of the stick refer to the sections of emulsion analyzed.

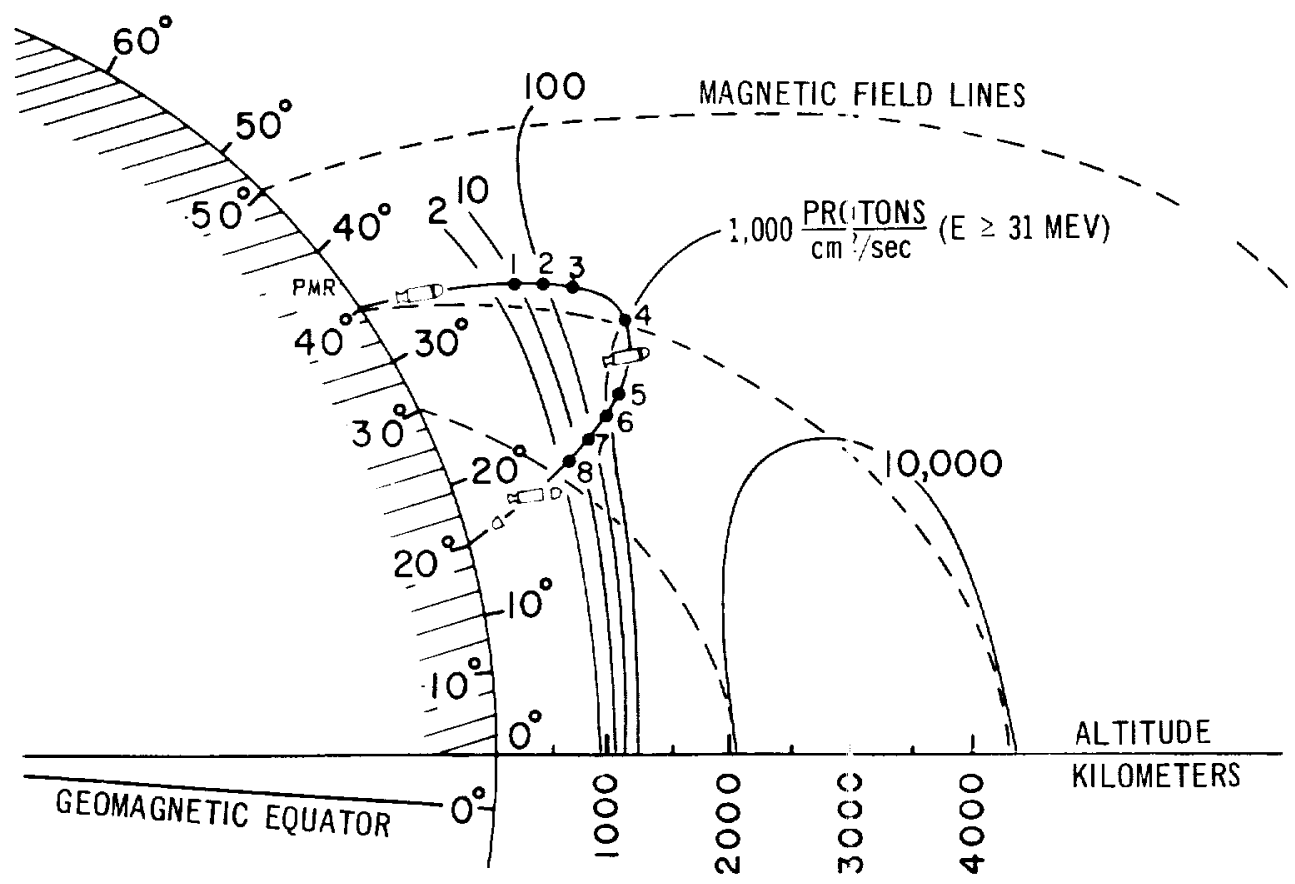

Figure 2 - Meridian section of the earth showing the trajectory of the rocket payload relative to the magnetic field and the radiation belt 
Table 1 - Summary of Results

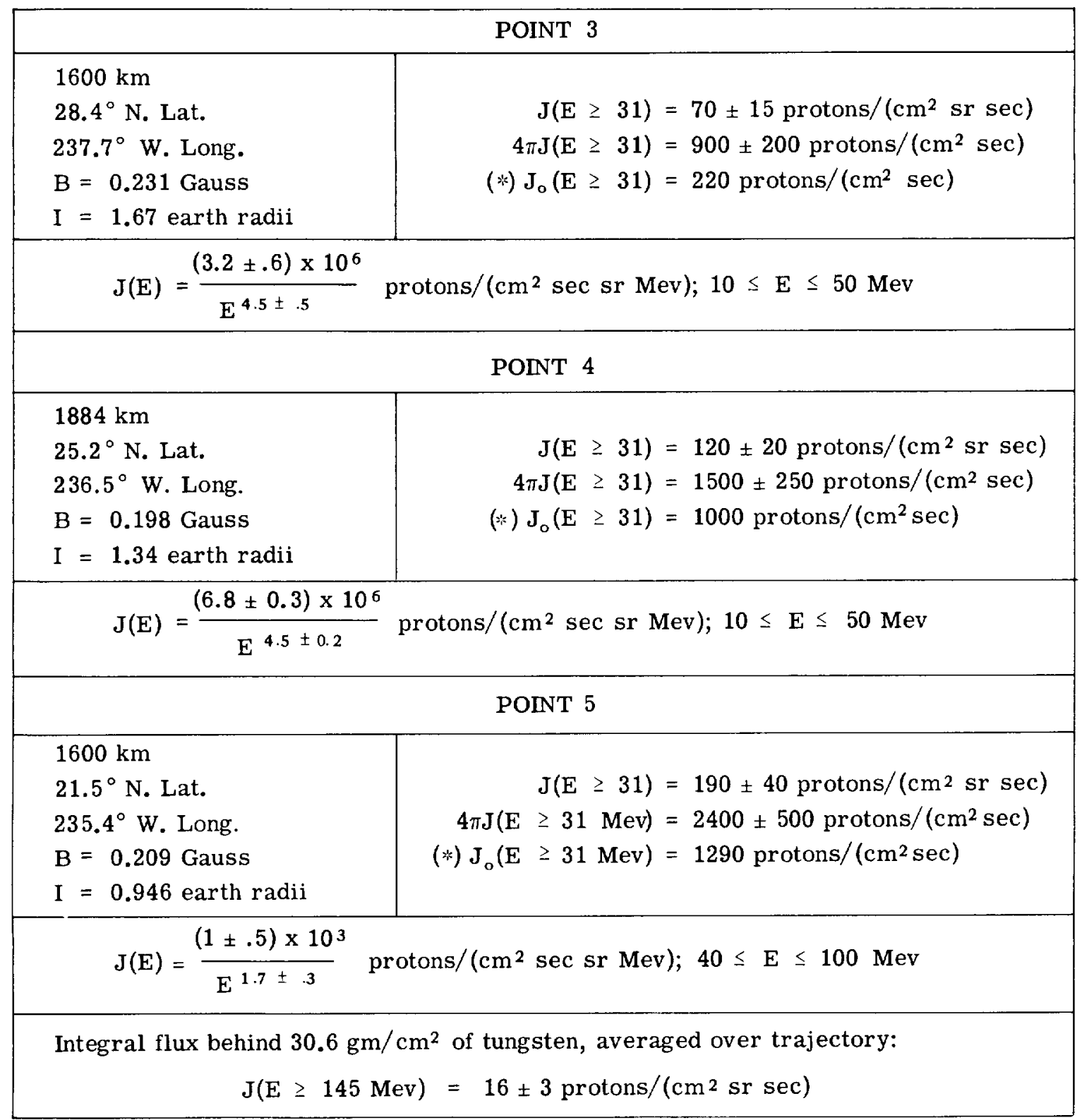

*Integral omnidirectional proton flux at point based on satellite data (Reference 7).

A "line scan" was made around the peripheries of the emulsions, approximately 500 microns from the edge; blackening prevented scanning closer to the edge. All particles whose tracks, at the scan line, were within \pm 25 degrees of a radius vector and within \pm 45 degrees of the plane of the emulsion were recorded. The portion of the emulsion not exposed at the port was also scanned, with the same criteria, to obtain an integral flux averaged over the entire flight and also a background correction for the other sections of the stack. 
To obtain the differential energy spectrum, all particles which had a possible residual range in the emulsion at the scan line of at least $2.5 \mathrm{~mm}$ were followed until they ended, interacted, or left the stack. The energy and mass of each of these particles was then determined from range, ionization, and scattering measurements.

\section{DISCUSSION OF RESULTS}

In the analysis to date, no evidence has been found for particles other than protons crossing the scan line. However, the mass measurements do not rule out a small component of singly-charged particles heavier than protons. The thickness of the blackened edge of the emulsion varies around the periphery of the stack and is consistent with the stopping in the emulsion of electrons with energies up to about $600 \mathrm{kev}$. There is a high background of grains extending another 500 microns inward from the black edge and possibly due to the presence of soft $\mathrm{X}$-rays.

The number of protons with energies greater than $100 \mathrm{Mev}$ crossing the scan lines was approximately equal to the number in the background area in the same energy range. Therefore it is not possible, with the statistics available at this time, to make reliable measurements of the spectrum above about $100 \mathrm{Mev}$.

The spectra obtained at the three points analyzed are shown in Figure 3 . Three things are apparent from these results:

1. At the higher latitudes, the slope of the energy spectrum below $40 \mathrm{Mev}$ is very steep when compared to predictions from conventional neutron albedo theory (References 3,4 and 5 ).

2. The shape of the spectrum and the flux both chanire with latitude; points 3 and 5 are at the same altitude and geomagnetic longitude.

3. At a comparable position in the belt (Point 5) the flux and the shape of the spectrum agree with previous data (Reference 1).

A change in the slope of the spectrum at higher energies with a change in latitude has been predicted by Singer (Reference 6) on the assumption that the maximum energy of the trapped protons decreases with altitude owing to the bre:tkdown of the adiabatic invariance of the magnetic moments of the trapped particles. It is also possible that the change in the spectrum at lower energies is due either (1) to solar protons trapped in the geomagnetic field or (2) to protons produced by the decay of albedo neutrons arising from solar proton interactions over the polar cap. In either case there would be a preferential trapping of low energy particles at the higher latitudes; but it is not clear which of these mechanisms is responsible for the change in the spectrum. A further study of these 

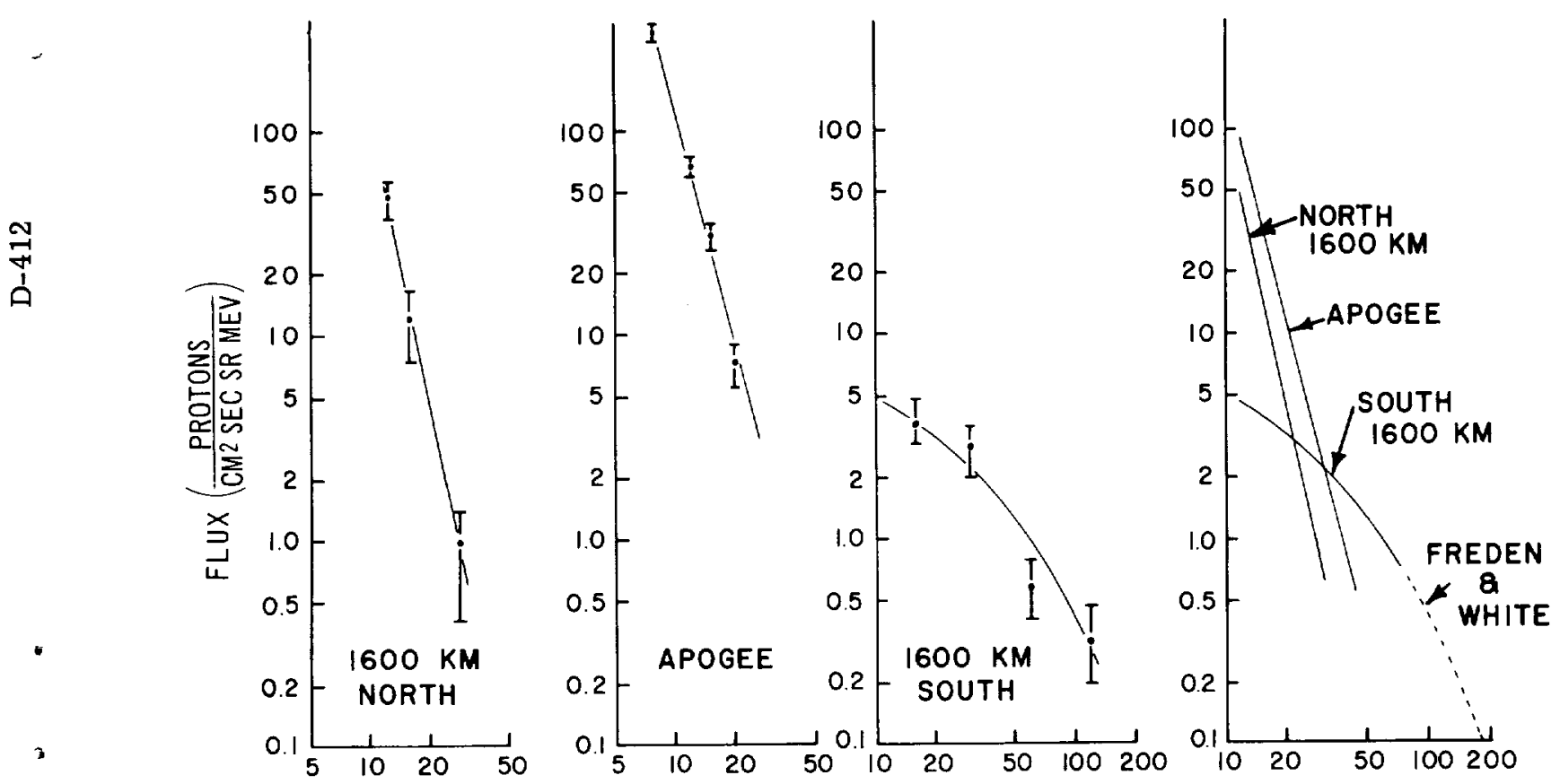

KINE TIC ENERGY (MEV)

Figure 3 - The differential energy spectra abtained at points 3, 4, and 5 on the trajectory, together with the three spectra superimposed for purposes of comparison

emulsions will be made to determine the maximum energy at which protons can be trapped at a given point. Additional flights are scheduled for late 1961 to determine whether there is a time variation. If the low energy protons are associated with solar activity, their flux should decrease as the number of solar proton events per year decreases during the solar cycle. Changes in the spectrum after a large solar flare have been reported by Armstrong and Heckman (Reference 2).

The unidirectional integral flux, $\mathrm{J}$, for protons with $\mathrm{E} \geq 31 \mathrm{Mev}$ is given in Table 1. In order to compare this flux with the satellite measurements, the assumption has been made that the flux is isotropic; therefore the integral omnidirectional flux can be obtained by multiplying $J$ by $4 \pi$. The values obtained by this method are listed in Table 1 with the best available value of the flux at the three points based on the analysis of the satellite data (Reference 7). The quoted errors are large because of the effects of the background on the integral flux; and the agreement is quite good considering these errors. The omnidirectional flux computed by assuming isotropy is consistently higher than the omnidirectional flux at all three points, indicating that the assumption is not valid.

The rocket nose cone was spin-stabilized nearly parallel to the magnetic field lines, and the unidirectional flux in the emulsion was measured primarily at angles near 90 degrees with respect to the field lines. At low altitudes, where there is a high density of mirror points, the flux should not be isotropic but should be higher at 90 degree angles, as was indeed observed. 


\section{ACKNOWLEDGMENTS}

The solution of the problems associated with the exposure and recovery of emulsions at $1880 \mathrm{~km}$ required outstanding and unique contributions from a number of people. These

efforts are gratefully acknowledged by the authors. In particular, the flight would not have been successful without the work, over and above his normal responsibility, of Project Engineer Charles E. Campbell, of the Goddard Space Flight Center.

\section{REFERENCES}

1. Freden, S.C. and White, R.S., "Protons in the Earth's Magnetic Field," Phys. Rev. Letters 3(1):9-11, July 1, 1959

2. Armstrong, A.H., Harrison, F.B., Heckman, H.H., and Rosen, L., "Charged Particles in the Inner Van Allen Radiation Belt," J. Geophys. Res. 66(2):351-357, February 1961

3. Freden, S.C., and White, R.S., "Particle Fluxes in the Inner Radiation Belt," J. Geophys. Res. 65(5):1377-1383, May 1960

4. Singer, S.F., "Trapped Albedo Theory of Radiation Felt," Phys. Rev. Letters 1(5):181183, September 1, 1958

5. Hess, W.N., "Van Allen Belt Protons from Cosmic-IRay Neutron Leakage," Phys. Rev. Letters 3(1):11-13, July 1, 1959

6. Singer, S.F. "On the Nature and Origin of the Earth's Radiation Belts," in "Space Research," Proc. 1st Internat. Space Science Sympos., Ed. by H. Kallman Bijl, Amsterdam: North Holland Publ. Co., 1960, pp. 797-820

7. McIlwain, C.E. and Van Allen, J.A., State University of Iowa, Private Communication 
